\title{
XII.
}

\section{Ein Vorschlag zur Radicalbehandlung des angeborenen Leisten bruches beim Mann.')}

\author{
Von \\ Dr. Carl Lauenstein, \\ Oberarzt des Seemannskrankenhauses und dirig. Arzt der chirurg. Abtheilung des Diakonissen- \\ hauses Bethesda za Hamburg.
}

Die vielfach besprochene Frage der Radicaloperation des angeborenen Leistenbruches harrt noch einer übereinstimmenden Antwort der Chirurgen.

Bei der Schwierigkeit der Beurtheilung, ob ein bestehender Leistenbruch angeboren sei, möchte ich hier nur diejenige Bruchart beriibren, welche mit mangelhaftem Descensus testiculi vergesellschaftet, sicher als angeboren zu erkennen ist.

Für diesen Bruch, bei dem eine stumpfe Loslösung des Hodens und Samenstranges fast immer unmöglich ist, stehen der Radicalbehandlung - abgesehen hier von der Schwalbe'schen Alkoholinjection - zwei Wege offen. Auf dem einen, von Kraske-Volk$\operatorname{mann}{ }^{2}$ ) zuerst betretenen, auf dem ihnen seitdem manche Chirurgen gefolgt sind, opfert man den in seiner Existenz durch die Operation ohnehin gefährdeten Hoden, um den Verschluss des Leistenkanals desto sicherer zu erreichen; auf dem anderen schont man den Hoden, indem man ihn, da er nicht ablösbar ist, mit einem Stück des Processus vaginalis, resp. des Bruchsackes aus diesem herausschneidet, verschliesst den Leistenkanal nach einer der üblichen Methoden und kann obendrein nach Schüller's ${ }^{3}$ ) Anweisung versuchen, den Hoden in die leergebliebene Scrotalhälfte zu "transplantiren".

1) Mittheilung am wissenschaftlichen Demonstrationsabend des neuen allgemeinen Krankenhauses vom 12. October 1889.

2) Kraske, Ueber die Radicaloperation bei angeborenen Leistenbrüchen der Männer. Centralblatt für Chir. 1882. Nr. 26.

3) Ueber die Transplantation des Leistenhodens in das Scrotum. Centralbl. für Chir. 1881. Nr. 52. 
Ueber den Erfolg dieses letzteren Bestrebens liegen in der Literatur bisber nur ganz vereinzelte Mittheilungen vor.

Mir selbst hat die S c bäller'sche Operation, das Freilegen durch Eröffnung des Processus vaginalis, Herunterziehen und Einnähen des Hodens in die betreffende Scrotalhälfte in einem Falle keinen bleibenden Erfolg geliefert, da schon wenige Wochen nachher der Hoden wieder an seiner früheren Stelle lag. Diesen Misserfolg bin ich geneigt darauf zuruckzuftihren, dass die Spitze des Scrotums kein fester Punkt ist, ganz abgesehen von den ubrigen dabei in Betracht kommenden Punkten, wie z. B. der Kürze des Samenstranges.

In der letzten Zeit vor die Frage der Radicalbehandlung des angeborenen Leistenbruches gestellt, habe ich mich dafir entschieden, den Hoden mitsammt dem Processus vaginalis in die Bauchhöhle zu verlagern, und glaube, dass vielleicht dieser Vorschlag dazu beitragen kann, die Schwierigkeit der Frage von der Ablösung des Samenstranges und der Behandlung des Hodens in solchen Fällen wesentlich zu verringern, wenn nicht ganz zu beseitigen.

Gerade weil wir über die Leistungsfähigkeit des oft in seiner Entwicklung gehemmten Hoden ts Bestimmtes wissen, so ist der Widerstand des Chirurgen gegen die Castration gerechtfertigt. Vorausgesetzt ferner, dass der Verlagerung des Hodens in die Bauchhöhle principielle Bedenken nicht entgegenstehen, so erfullen wir durch dieselbe die beiden Cardinalforderungen, welche in der Discussion über eine erfolgreiche Radicaloperation des angeborenen Leistenbruches beim Mann aufgestellt worden sind. Wir erhalten den Hoden und beseitigen trotzdem aus dem Leistenkanal den Samenstrang, dieses Organ, welches nach den ibereinstimmenden Anschauungen der Chirurgen ${ }^{1}$ ) den vollständigen Verschluss der Bauchwand binderte und dadurch die Bestrebungen der Radicaloperation wesentlich erschwerte.

Da der Vorschlag, welchen ich hiermit den Fachgenossen unterbreite, sich aus zwei durchsichtigen Factoren - 1. der Verlagerung des Hodens in die Bauchböhle, und 2. dem vollständigen Nahtrerschluss des Leistenkanals - zusammensetzt, so zögere ich nicht, denselben mitzutheilen, obwohl ich nur 2 Fälle, und auch diese erst vor ganz kurzer Zeit operirt habe.

Ich vermuthe iberdem, dass mein Vorschlag nicht nur allen den

1) Siehe hierzu namentlich: Socin, Ueber Radicaloperation von Hernien. Langenbeck's Archì für klin. Chir. 1879. Bd. XXIV. 
Chirurgen, die für die Schonung des Hodens eingetreten sind, sondern auch gerade allen denen willkommen sein wird, welche als Vertreter der Nothwendigkeit der Castration in einzelnen Fällen die Entfernung des Samenstranges aus dem Leistenkanal bisher als Conditio sine qua non zur Ermöglichung eines sicheren Verschlusses desselben gehalten haben.

Der erste der beiden Fälle betrifft einen 8 jährigen Knaben, aus der Praxis des Herrn Dr. Mingramm hierselbst, welcher mir Anfang September d. J. wegen einer Hernie vorgestellt wurde. Ieh fand eine linksseitige äussere Leistenhernie, die sich in etwa Zweifingerlänge und Zweifingerbreite vom äusseren Leistenringe nach der linken Hodenhälfte zu erstreckte und sich deutlich vorbauchte. Der linke Hoden fehlte, und nur der rechte war an seinem normalen Standorte, ein Factum, das bisher noch nicht beachtet worden war. Beim Betasten des linken äusseren Leistenringes fühlte man hin und wieder eine kleine bewegliche Geschwulst, die aller Wahrscheinlichkeit nach der Hoden war.

Schon bevor dies Fehlen des linken Hodens bemerkt worden war, hatte der Vater des Knaben sich mehr für die Radicaloperation, als für das Tragen eines Bruchbandes entschieden. Jetzt musste man das Tragen eines Bruchbandes unbedingt widerrathen, und es kam nur die Operation in Frage. Ueber die Chancen einer solchen befragt, musste ich es unentschieden lassen, ob ich den Hoden wtirde erhalten können. Ich konnte jedoch verstehen, dass für den Vater, der nur den einzigen Sohn hatte, diese Frage von grosser Wichtigkeit war. Indem er mir schliesslich als seinen Hauptwunsch die Beseitigung der Hernie bezeichnete, liess er mir in Bezug auf den Hoden vollkommen freie Hand, so zu handeln, wie es mir am zweckmässigsten scheinen würde.

Die 0 peration wurde am 17. September 1889 unter guitiger Assistenz der Herren DDr. Mingramm und Jentz ausgeführt. Von dem uiber die Bruchgegend geführten Längsschnitt aus liess sich der Bruchsack leicht und ohne Verletzung von dem umgebenden Gewebe frei machen. In ihm lag beweglich der Hoden, der sich etwas kleiner als der normale rechte anfühlte. Nachdem ich die Isolirung des Bruchsackes auch im Bereiche des Leistenkanals beendigt hatte, trennte ich den Macewenschen Vorschriften für seine Methode der Radicaloperation entsprechend durch Eingehen mit dem Finger in der Umgebung des inneren Leistenringes noch etwa $1 \mathrm{Cm}$. weit das Peritoneum parietale von der Innenfläche der Bauchwand $a b$, verschob den Hoden durch Streichen mit den Fingern in die Bauchhöhle, legte dann von der Bruchsackspitze an die Macewe n'sche Längsschntirnaht an und zog, indem ich in der Gegend des Halses den Catgutfaden in die Bauchhöhle und etwa 11/2 Finger breit oberhalb des Leistenkanals von innen nach aussen durch die Bauchdecke hindurchführte, durch $\mathrm{Zug}$ an diesem Faden den sich zusammenfaltenden Brucbsack in die Bauchböhle, derart, dass der Hoden auf dem sich hinter dem Annul. inguin. int. formirenden Kissen zu innerst zu liegen kam. Somit war die Bauchhöhle nicht weiter eröffnet worden, als durch die Stiche der die Wände des Brnchsackes treffenden Naht. Ich verschloss 
dann in der von Macewen empfohlenen Weise den Leistenkanal mit 2 Matratzennähten aus dickem Catgut, welche wie eine Klappe die innere Wand gegen die änssere befestigten, und nähte noch obendrein die Pfeiler des äusseren Ringes mit einer fortlaufenden Catgutnaht. Daruber fortlaufende Vereinigung der Haut mit Catgut ohne Drainage und Bedeckung der Wunde mit einem Gaze-Collodiumverband.

Es trat ungestörte Heilung per primam ein, ohne Fieber und ohne eine Spur von Secretion. Der Knabe, welcher jetzt in der 5. Woche nach der Operation und vollkommen munter ist, soll noch etwa 3 Wochen liegen, damit die Narbe ordentlich fest wird, ehe er aufsteht. 1)

Der zweite Fall war folgender:

Am 17. September d. J. wurdo der 33 jährige Seemann L. mit der Diagnose einer irreponiblen Hernie in das Seemannskrankenhaus aufgenommen. Er war 3 Tage zuvor auf dem Schiffe aus 5 Fuss Höhe rittlings in ein Boot gefallen und hatte sich dabei den Hoden gequetscht und gleichzeitig, wie er sagte, den Bruch zugezogen. Er gab an, dass er bis dahin stets gesund gewesen sei und nie einen Bruch gehabt habe. Bei der Aufnahme war die rechte Scrotalhälfte umfangreich mit Blut unterlaufen und etwa um das Dreifache gegentiber der linken, welche den normal grossen Hoden enthielt, vergrössert. Von der Gegend des rechten Leistenkanals bis in die rechte Scrotalhälfte hinein erstreckte sich eine mehrere Faust dicke, glatte, prall-elastische, aber nicht fluctuirende Geschwulst, welche bei der Percussion leeren Schall bot, während nach dem Leistenkanal zu der Schall tympanitischen Beiklang bekam. Rechts liess sich ans der Geschwulstmasse ein Hoden weder für Gesicht, noch Gefühl abgrenzen. Eine Reposition gelang nicht, wurde aber, wie ich sagen muss, auch in keiner Weise forcirt.

Da keine Incarcerationserscheinungen bestanden, Patient auch nicht fieberhaft war, so warteten wir einen vollen Tag ab.

Operation am 19. Sept. unter Assistenz der Herren DDr. Jentz und $\mathrm{Schabbel}$, im Beisein des Generalarztes der dänischen Marine Dr. Berg. Durch einen von der Gegend des Leistenkanals bis zur Hodensackspitze verlaufenden Schnitt wurde die Geschwulst freigelegt. Ich gelangte nach Durchtrennung des subcutanen Fettes auf einen weissglänzenden, straff gespaunten Sack, nach dessen Eröffnung sich als Inhalt der grösste Theil des Omentum majus zeigte, das nach der Mitte zu und nach abwärts mit der Innenfläche des Bruchsackes vielfach verklebt war. Als das Netz etwas gelöst war, zeigte sich noch ein anderer Inhalt, der bei genauerem Nachsehen als der rudimentäre Hoden mit seinem Samenstrang erkannt wurde. Letzterer lag flächenhaft der hinteren Wand des Bruchsackes an, während der Hoden etwa in der Mitte der Länge des Bruchsackes lag. Derselbe war etwa halb so gross als der normale Hoden, dabei von normaler, weissgrauer Farbe. Da es mir trotz Erweiterung des

1) Anmerkung bei der Correctur. Der Knabe ist nach Verlauf von 8 Wochen aufgestanden, besucht jetzt die Schule wieder und ist den ganzen Tag ausser Bett. Der Leistenkanal ist fest geschlossen, der Knabe trägt kein Bruchband und hat keinerlei Beschwerden. 
äusseren Leistenringes durch einen Scheerenschnitt nicht gelang, die im Leistenkanal stark eingeschnürte Netzmasse zu reponiren, so trug ich sie im Bereiche des Bruchsackhalses nach sorgfältiger Anlegung von 4 Massenligaturen ab und versenkte den Stumpf, dessen Wundfläche ich noch durch eine fortlaufende Catgutnaht versichert hatte, in die Bauchhöhle. Ich nahm mir nun vor, in ähnlicher Weise wie im vorigen Falle vorzugehen, musste jedoch wegen bestehender fester Verwachsungen hier die Isolirung des Bruchsackes theilweise mit dem Messer machen. Am äusseren Leistenring stiess die Isolirung insofern auf Schwierigkeiten, als der Bruchsackhals eine noch deutlich nachweisbare Hülle von der Fascia superficialis trug, welche in etwa Fingerbreite vom Annul. inguin. ext. sich an die Bauchdecke inserirte und daher circulär durchtrennt werden musste. Im Bereiche des Leistenkanals war der Bruchsack von einer Dünnheit, dass er offenbar nur aus Peritoneum bestand, doch gelang die Lösung bis uber den inneren Ring hinaus bei vorsichtigem Verfahren in erwünschter Weise. Einen Theil des hämorrhagisch unterlaufenen und mit Fibrinauflagerungen bedeckten Bruchsackes trug ich ab und formirte durch Nahtverschluss einen etwa um die Hälfte verkleinerten Bruchsack. Unter Beachtung, dass die Längsschnürnaht in gehöriger Entfernung von Hoden und Samenstrang gefiuhrt wurde, zog ich ganz wie im vorigen Falle den Bruchsack in die Bauchhöhle, nachdem ich den Hoden vorangeschoben hatte. Die Wände des Leistenkanals wurden durch $3 \mathrm{Ma}$ tratzennähte von dickem Catgut verschlossen, die Pfeiler des äusseren Ringes noch durch eine fortlaufende Naht vereinigt, während die Hautwunde wegen der Dicke des Fettlagers noch durch 2 Reihen fortlaufender Nähte geschlossen werden musste.

Nach der Operation trat eine sich allmählich herausbildende, etwa eigrosse, unter mehrtägigem mässigen Fieber verlaufende Nekrose im Bereiche der rechten Scrotalhälfte ein, von der es unentschieden bleiben musste, ob der angewandte Collodiumverband oder die voraufgegangene Quetschung Schuld daran war. Jetzt ist Patient schon lange fieberfrei und erfreut sich völligen Wohlbefindens. Der Serotaldefect granulirt gut und verkleinert sich zusehends. Patient soll ebenfalls noch mehrere Wochen das Bett hliten. ${ }^{\text {) }}$

Als wir denselben nach der Operation genauer über seine Hodenverhältnisse ausfragten, gab er an, dass er von Geburt an immer nur einen, und zwar linksseitigen Hoden gehabt habe.

Das Gewicht des abgetragenen Netzes betrug 11/2 Pfund.

Das Operationsverfahren, welches ich in diesen beiden Fällen eingeschlagen habe, ist hervorgegangen aus dem Macewen'schen Verfahren der Radicaloperation des Leistenbruches, dessen ich mich in der letzten Zeit bei der Behandlung von Leistenhernien ausschliesslich bedient habe. Macewen selbst ist zu dieser Consequenz, welche

1) Anmerkung bei der Correctur. Wegen Eiterung in den Bauchdecken vor dem äusseren Leistenrivg wurde eine erneute Spaltung der Weichtheile nothwendig. Jetzt bestehen aber ungestörte Wundverbältnisse, und die Heilung des kaum noch zweifingergliedlangen Wundspaltes steht nahe bevor. 
248 XII. Lauenstein, Zur Radicalbeh. des angebor. Leistenbruches beim Maun.

ich ans seiner Operationsmethode gezogen habe für den angeborenen Leistenbruch, nicht gekommen; vielmehr pflegt er so zu verfahren, dass er den Samenstrang mitsammt dem Hoden durch zwei parallele Längsschnitte aus dem Processus vaginalis peritonei excidirt, um dann den Rest des letzteren durch die Naht wieder zu einem Sack zu formiren und nach seiner originellen Idee als Kissen hinter, resp. vor den inneren Leistenring $z u$ lagern.

Kurz resumirt, ermöglicht das von mir in obigen 2 Fällen geubte und hiermit empfohlene Verfahren die Erhaltung des Hodens und den vollständigen Verschluss des Leistenkanals - Letzteres in einer Weise, wie man es bisher beim Mann nur unter Zuhülfenahme der Castration, ubrigens aber nur bei dem Leistenbruche des Weibes (Diverticulum Nuckii) auszuftuhren im Stande war.

Bei der Ausführung der Operation wird man selbstverständlich Acht geben milssen, dass nicht Samenstrang und Hoden durch das Zusammenfalten und Hereinziehen des Bruchsackes in die Bauchhöhle in ihrer Ernährung beeinträchtigt werden. Namentlich wird man hierauf bei der Anlegung der Schnürnaht Rücksicht zu nehmen haben, indem man sie je nach den Verhältnissen des einzelnen Falles nur von der Spitze des Bruchsackes bis an den Hoden heranführt, oder aber sie in möglichster Entfernung $n a c h$ aussen vom Hoden und Samenstrang anlegt. Da letzterer sich im Leibe nach innen wendet, so darf die Naht keinenfalls an der inneren Seite gefiubrt werden.

Einen Nachtheil der Verlagerung des Hodens in die Banchhöhle unter Berücksichtigung dieser technischen Vorsichtsmaassregeln für das Organ selbst gegenüber einer Lage vor dem Leistenkanal vermag ich a priori nicht zu erkennen. Eher noch scheint mir, dass er in der Bauchböhle geschützter liegt, als ausserhalb derselben.

So gut, wie das Ovarium seine angestammte Lage in der Banchhöhle hat, mag auch der Hoden ausnahmsweise, sofern er uberhaupt nicht verkümmert ist, innerhalb der Bauchböhle functionstuchtig bleiben.

Hamburg, 16. October 1889. 